\title{
Operational SAR-based sea ice drift monitoring over the Baltic Sea
}

\author{
J. Karvonen \\ Finnish Meteorological Institute (FMI), Helsinki, Finland \\ Correspondence to: J. Karvonen (juha.karvonen@fmi.fi) \\ Received: 11 January 2012 - Published in Ocean Sci. Discuss.: 30 January 2012 \\ Revised: 4 June 2012 - Accepted: 12 June 2012 - Published: 16 July 2012
}

\begin{abstract}
An algorithm for computing ice drift from pairs of synthetic aperture radar (SAR) images covering a common area has been developed at FMI. The algorithm has been developed based on the C-band SAR data over the Baltic Sea. It is based on phase correlation in two scales (coarse and fine) with some additional constraints. The algorithm has been running operationally in the Baltic Sea from the beginning of 2011, using Radarsat-1 ScanSAR wide mode and Envisat ASAR wide swath mode data. The resulting ice drift fields are publicly available as part of the MyOcean EC project. The SAR-based ice drift vectors have been compared to the drift vectors from drifter buoys in the Baltic Sea during the first operational season, and also these validation results are shown in this paper. Also some navigationally useful sea ice quantities, which can be derived from ice drift vector fields, are presented.
\end{abstract}

\section{Introduction}

Ice motion is a very important ice parameter for ice navigation. It can be used to locate the areas of divergence and convergence, and also be used to locate compressive ice fields. In addition to the ice drift up to the current moment, the forecasting of the ice drift is very useful information for ice navigation.

We at FMI have developed a SAR-based sea ice drift estimation algorithm, which is based on phase correlation at two resolutions. The algorithm is now run operationally as part of the MyOcean, which is a part of the European Commission (EC) GMES (Global Monitoring for Environment and Security) program. The SAR-based ice drift can be used for validating ice models making forecasts of ice drift based on numerical weather prediction data. It can also be utilized in data assimilation into the ice models, and as an additional information source for SAR-based sea ice classification algorithms. The data used in the FMI Baltic Sea ice drift estimation are from both Radarsat-2 and Envisat ASAR instruments. Since April 2012, after the breakdown of ENVISAT, only RADARSAT data have been used. These instruments operate at $\mathrm{C}$-band (wavelength about $5 \mathrm{~cm}$ ). The ice drift fields for the Baltic Sea are computed for each overlapping SAR image pair with a temporal distance less than or equal to two days. The resulting ice drift or ice displacement vector fields are delivered in NetCDF format, and are freely available for all registered MyOcean users (for the product catalogue, see (MyOcean product catalogue, 2012)).

Motion estimation methods between two co-registered images can be based on block matching (the similarity is typically measured by different variations of cross-correlation, such as phase correlation applied in our algorithm), algorithms estimating optical flow from differential equations based on partial derivatives of the image signal, such as presented in Horn and Schunck (1981), and feature-based methods. In feature-based algorithms, first some local features are computed and these are then matched based on some criteria (e.g. feature correlations) instead of direct block matching. Correlation (cross-correlation) methods have been utilized in estimation of ice drift, e.g. in (Fily , 1987), where ice drift was estimated from SAR image pairs using correlation in multiple resolutions. Other examples of correlation-based ice drift estimation from SAR are the Radarsat Geophysical Processor System (RGPS) (Kwok , 1998) and the Arctic and Antarctic MyOcean SAR ice drift products (MyOcean product catalogue, 2012) by Danish Technical University (DTU). In the RGPS, the correlation approach has been coupled with a feature-based approach. Correlation-based ice drift estimation methods have also been studied for the Baltic Sea earlier, e.g. in Sun (1994). In this approach also the rotation is estimated by correlating the power spectra, i.e. in this sense this 
algorithm can be considered as a combination of a featurebased and a correlation method. Optical flow-based ice drift estimation has been studied, for example for the Baltic Sea in Sun (1996). A multiresolution phase correlation algorithm for sea ice drift estimation was presented in Thomas et al. (2004, 2008), and this algorithm has also been tested in the Bay of Bothnia. These experiments were reported in Berg and Eriksson (2010). Ice drift estimation based on passive microwave instruments has been studied, e.g. in Liu and Cavalieri (1998); Haarpaintner (2006); the previous is an example of a correlation method, and the latter is a more sophisticated approach based on a wavelet decomposition, i.e. analysis of wavelet coefficients in multiple resolutions. Passive microwave instruments typically have a large coverage, but their resolutions are coarse, typically several kilometers, and only low- or medium-resolution ice drift can be measured by these instruments. SAR instruments instead have a much higher spatial resolution. In ScanSAR mode, which combines multiple SAR beams into one wide swath, with a width up to 400-500 km (ENVISAT ASAR and RADARSAT-1/2), SAR instruments also have a good spatial cover, with a high resolution of about $100 \mathrm{~m}$.

Our algorithm is based on computing phase correlation in two resolutions: in a coarse resolution to estimate the largescale drift; and in a fine resolution to refine the coarse-scale estimates. Because phase correlation is a correlation between phase information, it is sensitive to edges in the image, and phase correlation methods are robust against gray level variation, e.g. due to different incidence angles in the two images, compared to the common cross-correlation. Phase correlation is also more robust against noise than common crosscorrelation. Using multiple resolutions enables detecting of larger ice drift in a high resolution defined by the the fine resolution. The main differences between the earlier phase correlation algorithm in Thomas et al. $(2004,2008)$ and our new operational algorithm are (1) our algorithm uses only two resolutions (coarse and fine); (2) our algorithm performs the motion estimation only for areas containing some SAR features defined by existence of edges; (3) our algorithm uses a quality measure to measure the reliability of a drift estimate instead of the correlation value; (4) our algorithm selects the final motion from multiple motion candidates defined by multiple correlation maxima; and (5) our algorithm applies the vector median filtering (Astola et al., 1990) to refine the estimation (to produce more coherent vector fields). According to our experience, the SAR frequency band does not play a major role in ice drift estimation. The same features (edges) are visible in L- (FMI has used ALOSPALSAR C-band data), C- (RADARSAT, ENVISAT ASAR) and X-bands (COSMO-SkyMed, TerraSAR-X). In some areas, there can be some features at L-band, which are not visible at X-or C-bands, and in these cases ice drift detection is possible only at L-band. These features are due to the better penetration capability of L-band and thus ability to see deeper within the ice cover than at C- and X-bands. C- and $\mathrm{X}$-bands mostly represent surface scattering only.

\section{SAR Preprocessing}

We use both Radarsat-2 and Envisat ASAR data for the Baltic Sea ice monitoring. We use the wide swath mode (WSM) data from Envisat ASAR and ScanSAR wide (SCW) mode data from Radarsat-2. These modes assemble wide SAR images from several narrower SAR beams, resulting in an image width of $400-500 \mathrm{~km}$, which is a suitable size for operational sea ice monitoring at the Baltic Sea scale. The SAR resolution in these modes is still $100-150 \mathrm{~m}$. In the ice drift estimation, only the horizontally transmitted and horizontally received $(\mathrm{HH})$ polarization channel is used. Radarsat-2 also has a dual-polarized mode in ScanSAR mode, i.e. also HV (horizontally transmitted, vertically received) channel is available. Envisat ASAR does not have this possibility, however. The typical temporal coverage, i.e. a SAR image acquisition using these two instruments over the same area in the Baltic Sea, ranges from a few hours to three days.

The SAR images are first calibrated to get the logarithmic backscattering coefficient values, which are presented in decibels. The backscattering coefficient, $\sigma^{0}$, describes the properties of the target area producing the backscatter of each SAR pixel. The $\sigma^{0}$ values are then linearly scaled to eight bits per pixel ( $8 \mathrm{bpp}$ ) images; the scaling interval is from $-35 \mathrm{~dB}$ to $0 \mathrm{~dB}$. The general calibration equations are

$\sigma^{0}=\frac{A^{2}}{K} \sin (\alpha)=\frac{I}{K} \sin (\alpha)$,

$\sigma^{0}(d B)=10 \log _{10}\left(\sigma^{0}\right)$.

$K$ is a SAR calibration coefficient, and typically given in the SAR metadata; $A$ is the SAR amplitude value, and $I=A^{2}$ is the SAR intensity value. $\alpha$ is the SAR incidence angle, which increases from the near range to far range, and typically varies about in the range of 20-50 degrees.

The 8 bpp SAR images are then rectified to Mercator projection, which is the projection used in nautical charts in the Baltic Sea. The rectification is performed using a fixed reference latitude $\varphi_{0}$ (latitude of true scale) of 61 degrees for $40 \mathrm{~min}$. This parameter has been selected for the compatibility with the FMI ice charting applications and software onboard Finnish ice breakers. After rectification, a land masking to mask off all the land areas is applied.

For some other purposes, such as ice type classification, we also apply an incidence angle correction (Makynen et al., 2002), which is based on an empirical linear relationship between mean sea ice backscattering and incidence angle value. This correction is, however, not necessary for the ice drift estimation. 
For the ice drift application, the two images are coregistered. This is performed using the georeferenced data of the rectified images. After co-registration, the common areas of the two images are cut, and these two cut images are the inputs of the ice drift algorithm.

\section{Algorithm}

The algorithm is based on phase correlation computed in two resolutions. In our approach, the phase correlation computation is performed for edged areas only, because these typically are the areas, where reasonable correlations can be computed. In smooth or random areas, the correlations are too low for reliable drift detection.

The edges are here detected using the Canny edge detection algorithm (Canny , 1986), but in practice most of the edge detection algorithms produce similar results. Typically, the edge detection is based on gradient magnitude in the images. After edge detection, we perform a filtering of the edges, filtering out small edge segments. An edge segment is here defined as a set of connected edge pixels, in the sense of the pixel's 8-neighborhood. All the edge segments smaller than a given size (an integer number of pixels) are removed from the edge image. A suitable size threshold for a fullresolution SAR edge image is five. The small edge segments are typically due to speckle and do not describe any actual SAR features.

The ice motion is determined for sampled data windows from the two images using phase correlation. The window size is $W \times W$; we have used $W=16$. The detection of the ice drift at each resolution is based on the phase correlation. To compute the phase correlation, the 2-dimensional fast Fourier transform (2-D FFT) is applied to the data windows sampled from the two co-registered images at the same location. Then FFT-coefficients of the two image windows are normalized by their magnitudes, and the FFT-coefficients of the two image windows are multiplied, and the inverse 2-D FFT is applied. The phase correlation array is computed from the the normalized cross power spectrum. The best matching displacement in a Cartesian (x,y) coordinate system is defined by the maximum of the phase correlation, here denoted by PC:

$$
\begin{aligned}
& (d x, d y)=\operatorname{argmax}_{(x, y)}\{\mathrm{PC}(x, y)\} \\
& =\operatorname{argmax}_{(x, y)}\left\{\mathrm{FFT}^{-1}\left(\frac{\left(X_{1}{ }^{*}(k, l) X_{2}(k, l)\right)}{\left|X_{1}{ }^{*}(k, l) X_{2}(k, l)\right|}\right)\right\},
\end{aligned}
$$

Because the FFT assumes the data to be periodic, a Gaussian window is applied to the data windows before the transformation. The drift is estimated in the row-column coordinate system in whole pixels at two resolutions. The displacement vector in the row and column coordinates is denoted by $(d r, d c)$. In practice, there often occur several correlation maxima which can be close to each other, and it is reasonable to use more than just one drift candidate for one window pair, and make the final decision only at the fine resolution level.

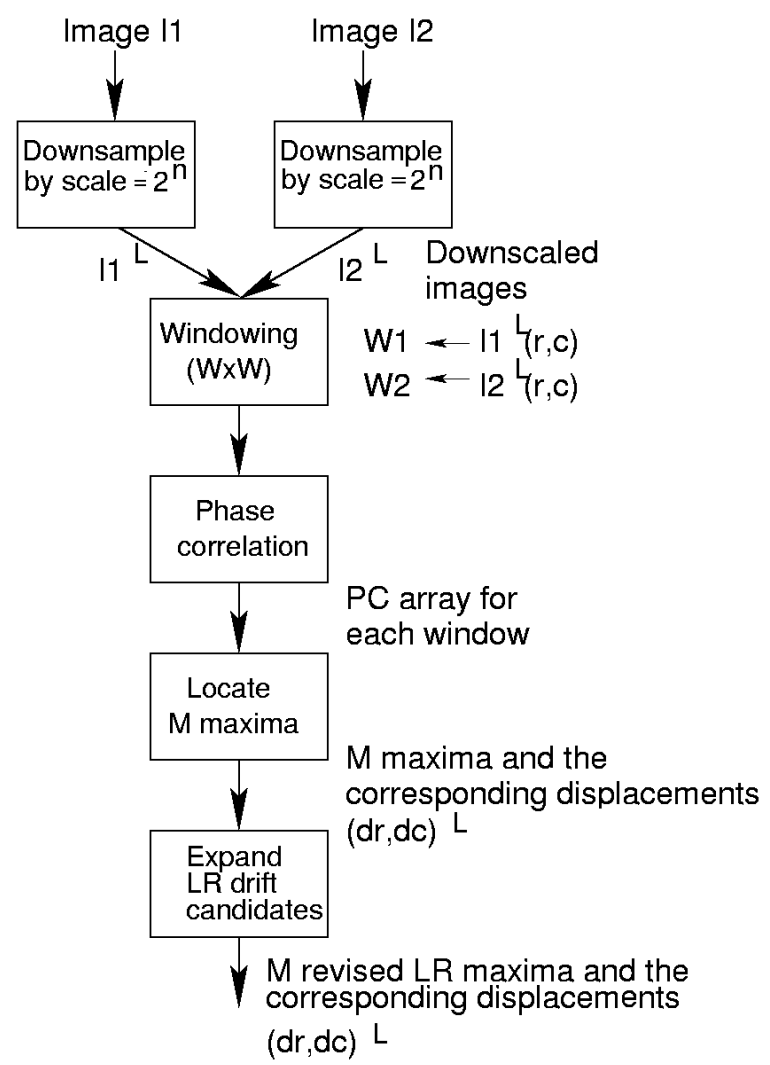

Fig. 1. The low-resolution part of the algorithm. The processing after down-sampling is shown only for one window pair in the diagram. In the algorithm, this is performed for each window pair with a grid step of $(\mathrm{W} / 2, \mathrm{~W} / 2)$ low-resolution pixels.

Two resolutions are used in this approach: first, coarse- or low-resolution (LR) data are used to locate the large-scale motion; then fine- or high-resolution (HR) data are used to refine the motion estimation. Simplified flow diagrams of the algorithm for the low-resolution and high-resolution parts are shown in the Figs. 1 and 2.

For the low resolution, the two co-registered images are first down-sampled to the given resolution. The downsampling rate is a power of two, say $R_{S}=2^{n-1}$. We have used $n=5$, corresponding to a down-sampling $R_{S}=16$. The two low-resolution images are generated by successively applying a half-band low-pass FIR filter designed for multi-resolution image processing (Biazzi et al., 1998). After down-sampling to the low resolution, we go through the image in steps of $W / 2$ in both row and column directions, and for each of the sampled locations, we make the two $W \times W$ sample windows, and locate $M$ phase correlation maxima. We have used the parameter value $M=12$ here. If the zero motion case $(d r, d c)=(0,0)$ is not included in the $M$ low-resolution drift values indicated by the maxima, it is included and the drift candidate with the lowest phase correlation is excluded. In the same way, the low-resolution 


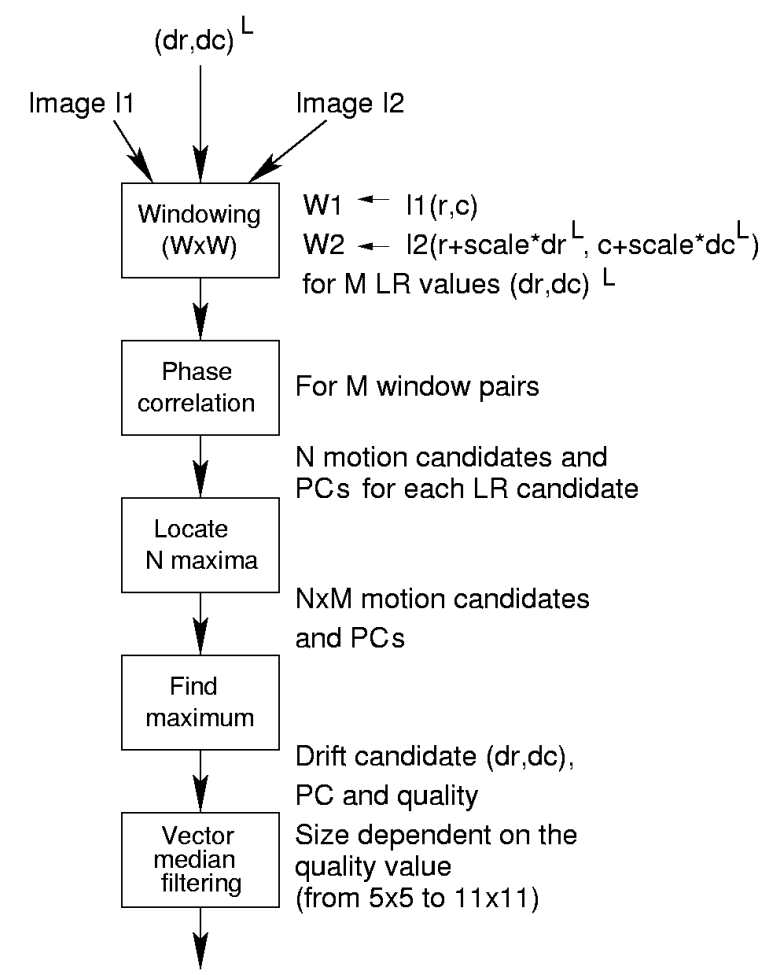

Ice drift (dr,dc), PC, and quality for each location

Fig. 2. The high-resolution part of the algorithm. The processing is shown here only for one window pair. In the algorithm, this is performed for each window pair with a grid step of $(\mathrm{W} / 2, \mathrm{~W} / 2)$ highresolution pixels.

drift candidates with the highest phase correlations from the neighboring low-resolution motion grid locations are copied to the list of $M$ candidates, if they are not included. This revised list of drift candidates and corresponding phase correlations for each image location is then delivered to the fineresolution processing.

At the fine-resolution level, all the displacements from the low-resolution level, scaled up by the down-sampling factor, are considered as potential low-resolution ice drift candidates, and the windows are sampled in a $W / 2 \times W / 2$ pixel grid from the full-scale first image (i.e. the step resolution is $800 \mathrm{~m}$ for $100 \mathrm{~m}$ resolution images). The sampling locations from the second image are defined by the $M$ scaled displacements from the low resolution. For each highresolution window pair, we also locate multiple drift candidates corresponding to $N$ highest maxima, thus resulting in $M \times N$ drift candidate values for each fine-scale location in the $W / 2 \times W / 2$ grid. In the current version of the algorithm, the drift candidate with the highest phase correlation at the fine-resolution level is selected as the final ice drift candidate. In another algorithm version, we have also performed some spatial filtering within a given spatial neighborhood among all the $M \times N$ candidates at each grid location before select- ing the final drift candidate. Some geophysical restrictions can additionally be used, e.g. not allowing too high local convergence values, which can be adjusted by a threshold value.

Finally, a vector median filtering (Astola et al., 1990) is performed with a given radius to obtain the final motion estimate. The size of the filtering is dependent on a quality index, and is varied from $5 \times 5$ to $11 \times 11$ points in the fineresolution motion grid. The algorithm can actually use either phase correlation or cross-correlation (this can be selected by the user), but we have used only phase correlation in the studies presented here and in the operational algorithm.

\subsection{Quality Measures}

The cross-correlations or the phase correlations are not very good measures of the quality of the estimates. This is due to the fact that there often also exist other high correlation values at different locations between two data windows. And if there are more than one correlation peak, it is difficult to determine which of these peaks corresponds to the actual motion and which are due to similarities spatially apart from each other in the images. Different quality indexes instead of phase or cross-correlation, taking into account the possible existence of multiple correlation peaks, have been studied at FMI. Here are some quality measures, which can be used. The sub-indexes of the phase correlation (PC) refer to the sorted (largest first, starting from one) phase correlation values for one SAR window pair:

$Q_{1}=\mathrm{PC}_{1}\left(1-\sum_{k=2}^{n} \frac{\mathrm{PC}_{k}}{\mathrm{PC}_{1}} D_{k}\right)=\mathrm{PC}_{1}-\sum_{k=2}^{n} \mathrm{PC}_{k} D_{k}$

$Q_{2}=1-\sum_{k=2}^{n} \frac{\mathrm{PC}_{k}}{\mathrm{PC}_{1}} D_{k}$

$Q_{3}=\mathrm{PC}_{1}-\mathrm{PC}_{2}$

$Q_{4}=\mathrm{PC}_{1} \times\left(\mathrm{PC}_{1}-\mathrm{PC}_{2}\right)$

$Q_{5}=\mathrm{PC}_{1} / N_{p}$

$Q_{6}=1.0-\left(\mathrm{PC}_{2} / \mathrm{PC}_{1}\right)=\frac{\mathrm{PC}_{1}-\mathrm{PC}_{2}}{\mathrm{PC}_{1}}$

$D_{k}$ are the pairwise distances of the lower ( $k$-th) maxima from the first maximum. In the quality measures not utilizing the distance of the maxima, the maxima in the neighborhood of the first maximum are excluded, because typically the phase- or cross-correlation is also high and near the 
peaks, but this only indicates the same peak value. $N_{p}$ is the number of $\mathrm{PC}$ peaks higher than or equal to $f_{c} \mathrm{PC}_{1}$. We have typically used $f_{c}=0.7$. This kind of quality index is much better criterion for including or excluding data than the phase or cross-correlation. Our current algorithm by default computes the quality measures $Q_{5}$ and $Q_{6}$.

\section{Operational products}

The products are computed using a window size of $16 \times 16$ pixels in the Mercator projection, and the computation step size is 8 pixels in both row and column direction, corresponding to about $800 \mathrm{~m}$ for $100 \mathrm{~m}$ resolution images. Also, the nominal product resolution is $800 \mathrm{~m}$. The values used in the products are the row- and column components of the motion, phase correlation and the quality values $Q_{5}$ at each $800 \mathrm{~m}$ grid cell. In the operational products delivered to MyOcean (MyOcean product catalogue, 2012), the drift components and the scaled quality value are re-sampled to latitudelongitude coordinate system with a similar resolution as in the Mercator coordinates. Bilinear interpolation for the vector components is used in the re-sampling. The values in the final product are given as U- (from west to east) and Vcoordinates (from south to north) in meters, i.e. the motion in pixels is multiplied by the local resolution. The local resolution $R_{L}$, in the Mercator projection, is computed as

$R_{L}=\frac{\cos \left(\varphi_{0}\right)}{\cos (\varphi)} R_{0}$.

$R_{0}$ is the true resolution at $\varphi_{0}-$ in our case $100 \mathrm{~m}$.

As a measure of product quality, we have used a scaled version of the quality measure $Q_{5}$ in the operational product. This value is delivered as part of the ice drift product for each grid point.

The quality measure $Q_{5}$ is converted to a scaled quality index $Q_{s}$ ranging from one to five using the following thresholds:

$$
\begin{aligned}
& Q_{5}<1^{-5} \rightarrow Q_{s}=0 \\
& 1^{-5} \leq Q_{5}<1^{-3} \rightarrow Q_{s}=1 \\
& 1^{-3} \leq Q_{5}<0.1 \rightarrow Q_{s}=2 \\
& 0.1 \leq Q_{5}<0.2 \rightarrow Q_{s}=3 \\
& 0.2 \leq Q_{5}<0.4 \rightarrow Q_{s}=4 \\
& Q_{5} \geq 0.4 \rightarrow Q_{s}=5
\end{aligned}
$$

The final product is converted into a NetCDF file, with georeferencing for the common area of each overlapping SAR image pair within a mutual temporal distance of two days.

\section{Applications}

We also compute some derived quantities from the ice drift; the most important quantities for modeling and navigation are shortly presented here. First, the mean ice drift velocity between a SAR image pair can be computed by dividing by the SAR acquisition time difference. Mean velocity is a useful value, for example, in ice model validation and assimilation. The mean ice velocity can be computed from the ice model ice drift velocities by means of numerical integration.

Other useful quantities are divergence, curl and shear, and quantities derived from these. Divergence $D(F)$ of a vector field $F=(d r, d c)$ is defined as

$D(F)=\nabla \cdot F$.

$d r$ and $d c$ are the motion in row and column directions. A simple discrete estimate for divergence $D$ at location $(i, j)$ is

$D(F)=\frac{1}{2}[d r(i+1, j)-d r(i-1, j)+$
$d c(i, j+1)-d c(i, j-1)]$.

The divergence (blue) and convergence (red) for the ice motion example in Fig. 4 are also shown in the vector field part of the figure.

Curl $C$ of a vector field $F$ is

$C(F)=\nabla \times F$.

And the corresponding discrete estimate for curl at $(i, j)$ is

$$
\begin{aligned}
& C(F)=\frac{1}{2}[d c(i+1, j)-d c(i-1, j)- \\
& d r(i, j+1)+d r(i, j-1)] .
\end{aligned}
$$

The direction of the curl vector is the direction of the normal of the vector field, and the sign of the curl indicates the direction (clockwise or counter-clockwise).

Shear $S$ for a vector $F$ field is

$S(F)=\sqrt{\left(\frac{\partial d c}{\partial c}-\frac{\partial d r}{\partial r}\right)^{2}+\left(\frac{\partial d c}{\partial r}-\frac{\partial d r}{\partial c}\right)^{2}}$.

In a discrete estimate, we replace the partial derivatives in Eq. 16 as follows:

$$
\begin{aligned}
\frac{\partial d c}{\partial c} & =\frac{1}{2}[d c(i, j-1)-d c(i, j+1)] \\
\frac{\partial d r}{\partial r} & =\frac{1}{2}[d r(i-1, j)-d r(i+1, j)] \\
\frac{\partial d c}{\partial r} & =\frac{1}{2}[d c(i-1, j)-d c(i+1, j)] \\
\frac{\partial d r}{\partial c} & =\frac{1}{2}[d r(i, j-1)-d r(i, j+1)] .
\end{aligned}
$$

After computing the discrete estimates for divergence and shear, the total deformation can then be computed as

$D_{T}(F)=\sqrt{S^{2}+D^{2}}$.

One practical application utilizing the ice drift estimation is locating fast ice. This can be done by finding the areas 


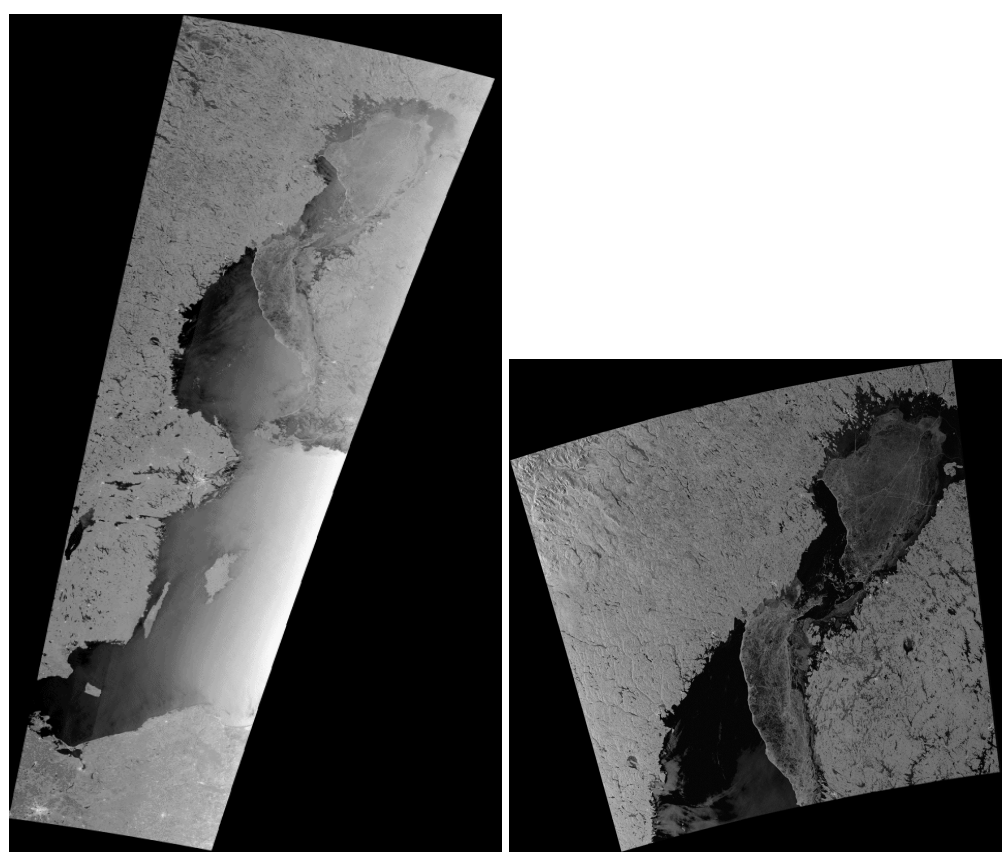

Fig. 3. SAR images from 3 April 2011 at 09:17:34 UTC (Envisat ASAR, CESA, left) and 3 April 2011 at 16:12:42 UTC (Radarsat-2, @MDA, right) over the Gulf of Bothnia.

where practically no ice motion has occurred within a relatively long time period. In the Baltic Sea, a suitable period for fast ice detection is two weeks or longer. An example of a two-week cumulative ice motion magnitude in the Baltic Sea can be seen in Fig. 5. Fast ice areas can be distinguished from this by applying a threshold, and labeling all the areas with a cumulative ice motion magnitude below the threshold as fast ice.

Some of the areas where ice compression occurs can also be estimated based on ice deformation indicators based on SAR ice motion, and these indicators are very useful in the validation of ice models trying to forecast ice compression for ice navigation.

Some statistics of the measures based on estimated ice motion can also be used to get at least some qualitative information on the ice thickness. From the total ice deformation computed over e.g. a period of two weeks, we can locate the areas of high deformation, which typically include thicker ice than areas of lower deformation.

One feature derived from the ice drift is the ice drift ratio, denoted by $R_{M}$ at each grid position:

$R_{M}(r, c)=\sum_{i=t_{o}}^{t_{n}}\left|\mathbf{f}_{i}(r, c)\right| /\left|\sum_{i=t_{o}}^{t_{n}} \mathbf{f}_{i}(r, c)\right|$

In the equation, $\mathbf{f}_{i}(r, c)$ is the ice motion vector at location $(r, c)$ at the moment $i$. In practice, the moment describes the drift between two adjacent SAR images. The sums are computed over the motion vectors from SAR image pairs during a certain time period, e.g. one week or two weeks. The sum in the numerator is the sum of all the drift magnitudes during the time period, and the sum in the denominator is the absolute value of the cumulative motion. $R_{M}$ is typically higher in the areas of thicker ice and lower in the areas of thinner ice. This can be explained such that, in the areas where this ratio is higher, the motion has not been in a certain direction, but the motion direction has been oscillating, and in the areas of low ratio the motion has been more in one direction. In the fast ice areas (black areas in Fig. 6), the ice thickness can quite accurately be estimated based on a thermodynamic ice model (Launiainen and Cheng, 1998), and if we also get a reliable ice thickness distribution from an ice model, we then can give some ice thickness estimates over the drift ice areas based on the drift statistics described. The deficiency of utilization of this kind of statistics is that it requires data over a time period of some weeks, and thus cannot take into account faster temporal ice dynamics. This information can, for example, be used to improve the initial ice thickness fields in an ice thickness estimation algorithm based on thermodynamic ice model and SAR data (Karvonen et al., 2008a,b). In Fig. 6, the total ice deformation, ice drift ratio $R_{M}$, and the mean level ice thickness (from FMI ice chart based on ice analysis from multiple data sources) for 17 March 2011 as an example is given. The thinner ice areas roughly correspond to the areas where the total deformation and the drift ratio are smaller, excluding the fast ice areas.

The main uses for the Baltic Sea ice drift estimates will be the ice classification algorithms (ice thickness, ice type 


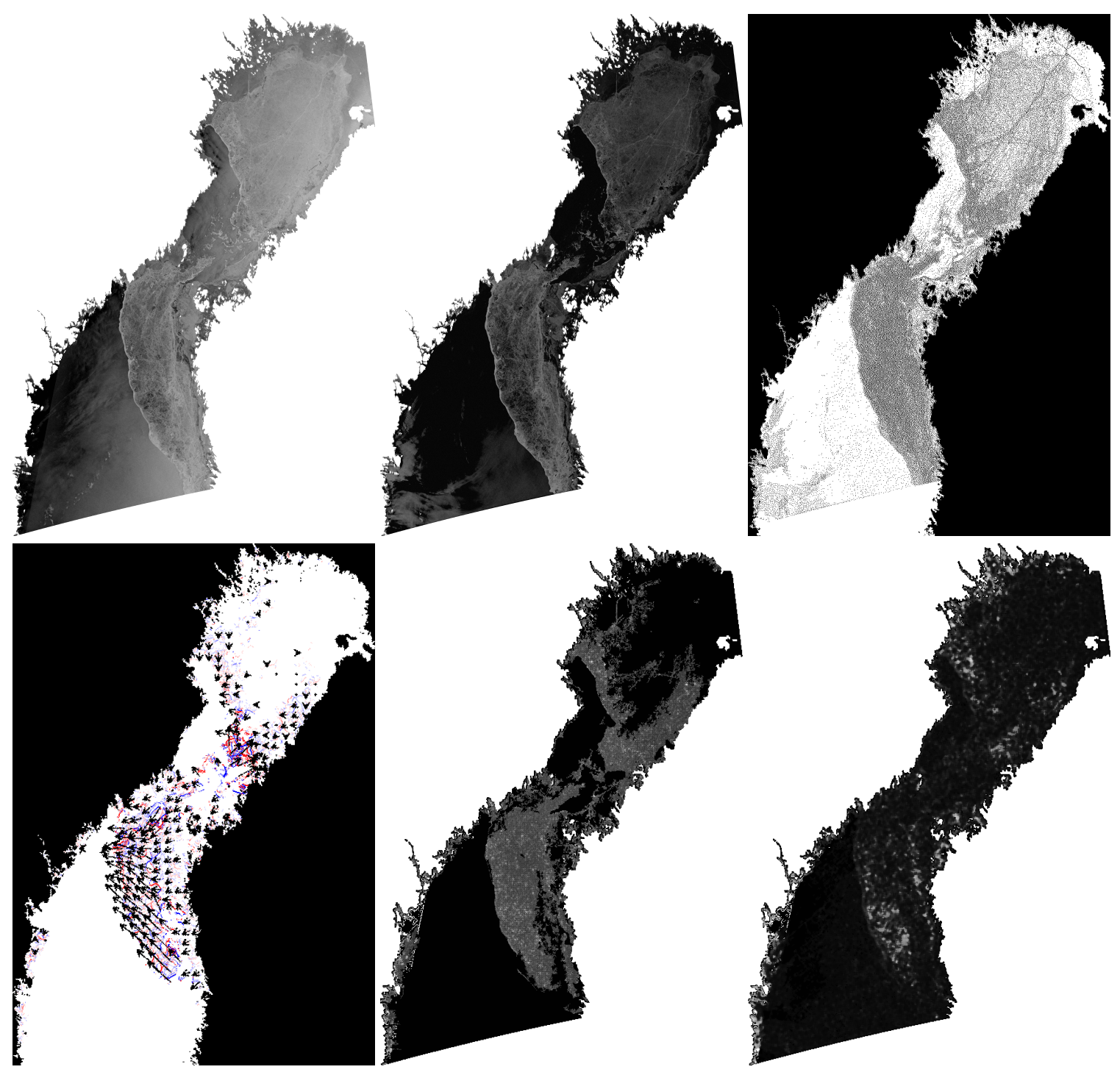

Fig. 4. The SAR images of Fig. 3 after co-registration and land masking (ASAR CESA, upper left, and Radarsat-2 @MDA, upper middle), and the located edges indicating the edged areas (edges shown in black, upper right). Ice motion vector field between the SAR acquisitions on 3 April 2011 at 09:17:34 UTC and 3 April 2011 at 16:12:42 UTC over the Gulf of Bothnia (lower left). For better visibility, the motion vectors are shown in a coarser grid than the nominal resolution and their lengths are scaled. The longest vector corresponds to a motion of $2.83 \mathrm{~km}$; the maximum motion in both row and column directions was $21100 \mathrm{~m}$ pixels. The scaled phase correlation (brighter tone indicates higher values, lower middle), and the scaled quality index $Q_{5}$ (brighter tone indicates higher values, right).

classification), validation of ice models, and data assimilation into ice models.

\section{Validation}

During the 2011 validation period, i.e. from 1 March to 26 April 2011, a total of 209 SAR-based ice motion estimates computed for the common areas of SAR image pairs with a temporal difference were used in the validation. 169 of these were classified as short drift category (buoy motion less than
$500 \mathrm{~m}$ ) and 40 measurements as long drift category (the rest of the data). The buoy trajectories during the test period are shown in Fig. 7.

The buoy locations were transmitted only once an hour, and the temporally nearest buoy locations to the SAR acquisition times were used in the comparison. This temporal inaccuracy naturally produced some inaccuracies. The scaled quality $Q_{s}$ for all the long drift data was two. For the short drift data, $Q_{s}$ varied in the interval 2-4. For the short drift data, only the motion magnitude was evaluated, because, in short motion estimates, the SAR registration errors can cause 


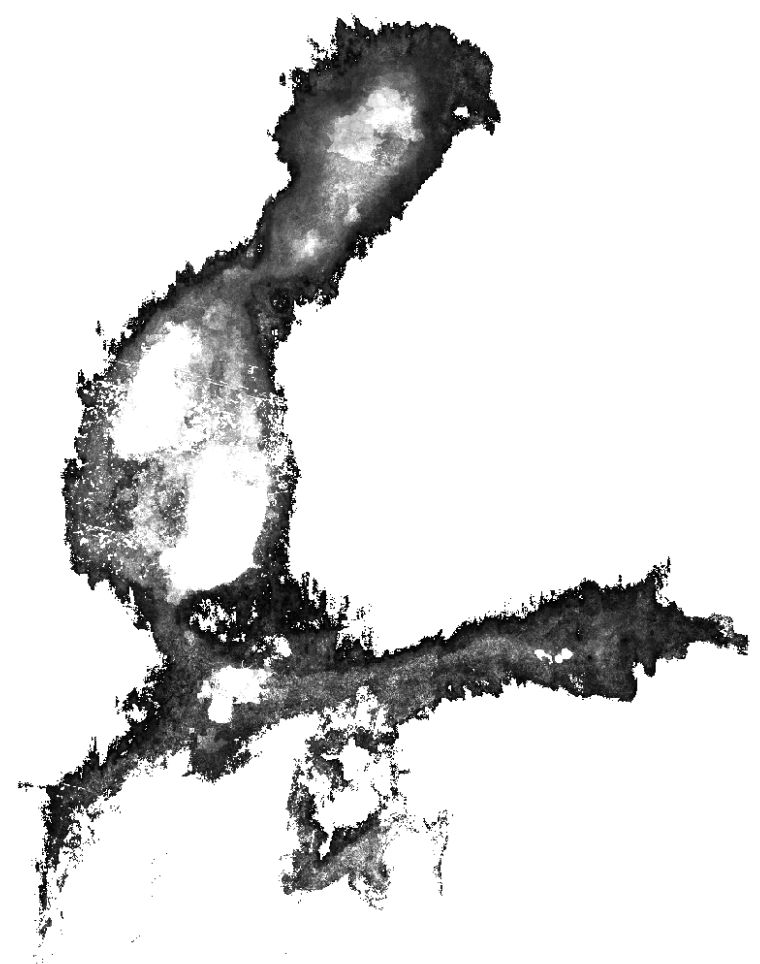

Fig. 5. Mean ice motion magnitude for two weeks before 5 March 2010 based on our SAR ice drift algorithm. Bright tones represent larger motion. The fast ice areas can be derived from these data. The bright areas in the southern parts of the Baltic Sea are open water. Open water has been masked off using the method presented in Karvonen et al. (2005) and set white in the figure.

large relative errors and defining the direction can become ambiguous. Also for short motion when computing motion in pixels, the direction is highly quantized increasing the error. For example, if the estimated drift is one pixel, it can be only in four directions (left, right, up, down) and thus the direction is quantized into four angles. For the long drift data, both the magnitude and direction were combined. The validation results are shown in Figs. 8-10.

The coefficient of determination, $R^{2}$, for the long drift data magnitude was 0.55 (with one obvious outlier removed, the outlier buoy motion is more than theoretically possible to detect using the applied algorithm parameters, i.e. about $18.1 \mathrm{~km}$ in diagonal direction), and the mean absolute direction difference was 15.8 degrees. For the short drift data, we computed the number of cases where the buoy motion was over $1000 \mathrm{~m}$, i.e. the magnitude error is already rather large (over $500 \mathrm{~m}$ ) and the estimated motion is short (i.e. less than $500 \mathrm{~m}$ ). This number was 11 of the 168 samples, i.e. $6.5 \%$. The mean absolute direction difference for the short motion was 75.8 degrees. Some computed error measures for the two cases are tabulated in Tables 1 and 2.
Table 1. Long motion error statistics: the magnitude errors (except the RMSE) are in meters.

\begin{tabular}{ccccc}
\hline Quantity & L1 err. & MSE & RMSE & bias \\
\hline Magnitude & 847 & 1333 & 0.15 & -470 \\
Angle & $15.8^{\circ}$ & $21.3^{\circ}$ & - & - \\
\hline
\end{tabular}

Table 2. Short motion magnitude error statistics (the errors are in meters).

\begin{tabular}{ccccc}
\hline Quality $Q_{s}$ & Samples & L1 err. & MSE & bias \\
\hline 2 & 139 & 842 & 1320 & 790 \\
3 & 24 & 144 & 178 & 130 \\
4 & 6 & 326 & 368 & 320 \\
\hline
\end{tabular}

We also computed the $L_{1}$ error and mean square error (MSE) for the long motion magnitude and angle, and for the short motion magnitude for each of the detected quality classes. In the long motion case, there was only one quality class $\left(Q_{s}=2\right)$. We also computed the bias (estimated magnitude-buoy magnitude), and it can be seen that the long magnitudes are underestimated and the short magnitudes are overestimated by the algorithm. The relative mean square error (RMSE) for the long motion magnitude was 0.15 and for the short motion magnitude 0.49 . The direction in the long motion case was estimated rather well. In comparison to visual interpretation for some image pairs, the motion estimated by the algorithm seemed to correspond to the visual interpretation.

For the small drift data, the magnitude mean absolute error was $842 \mathrm{~m}$ for the data with the scaled quality $Q_{s}=2(139$ samples), $144 \mathrm{~m}$ for the data with $Q_{s}=3$ (24 samples), and $326 \mathrm{~m}$ for the data with $Q_{s}=4$ (6 samples). It seems based on this small data set that the estimates are better; $Q_{s}$ higher than 2 gives better estimates, but the amount of data is still too small to make final conclusions on this. In general, the products show that the SAR-based ice drift estimates give useful estimates of the ice drift; especially the direction of the drift is estimated well. However, there exist some relatively large errors. We have not yet studied the locations of the erroneous measurements, but we assume them to be located close to edges of large drift and small drift areas. It can also be expected that the errors for the short drift data are higher, because the relative errors, due to e.g. image pair registration and the direction quantization, become larger. This can be seen especially in the value of the RMSE. Because the long drift directions are estimated rather well, and also it is rather well estimated whether the drift magnitude is short or long, these ice drift estimates are useful in validating ice models, especially after a visual inspection of the drift estimate data and the SAR images in parallel before the validation process, and leaving out the data that possibly do not 

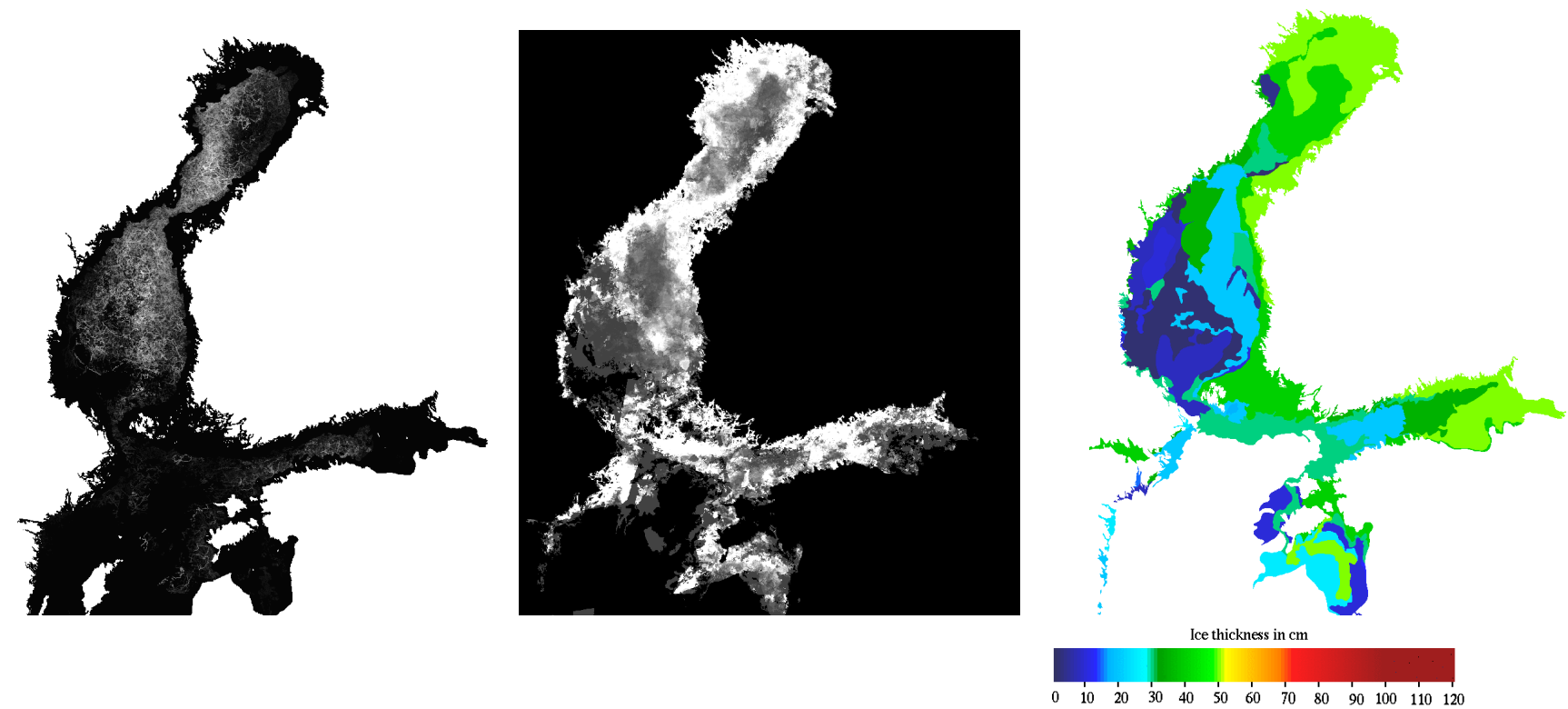

Fig. 6. Ice total deformation (left), ice motion ratio (middle), and the mean level ice thickness on 17 March 2011. The total deformation and ice motion ratio are computed over two weeks before $17 \mathrm{March}$, and the brighter tones indicate higher values.

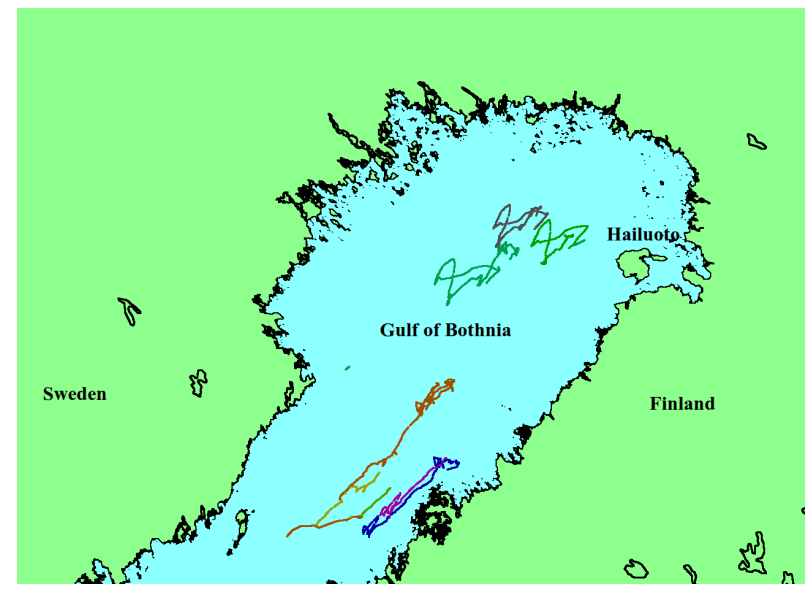

Fig. 7. Buoy tracks shown with different colors for each buoy.

correspond to the visual interpretation. We have also shown that this kind of data can successfully be used in improving SAR-based ice classification algorithms (locating fast ice and getting information on the degree of deformation).

\section{Conclusions}

We have developed an operational SAR-based sea ice drift algorithm, product generation and delivery chain at FMI. The products have been generated and delivered operationally during one Baltic Sea ice season, and also been validated

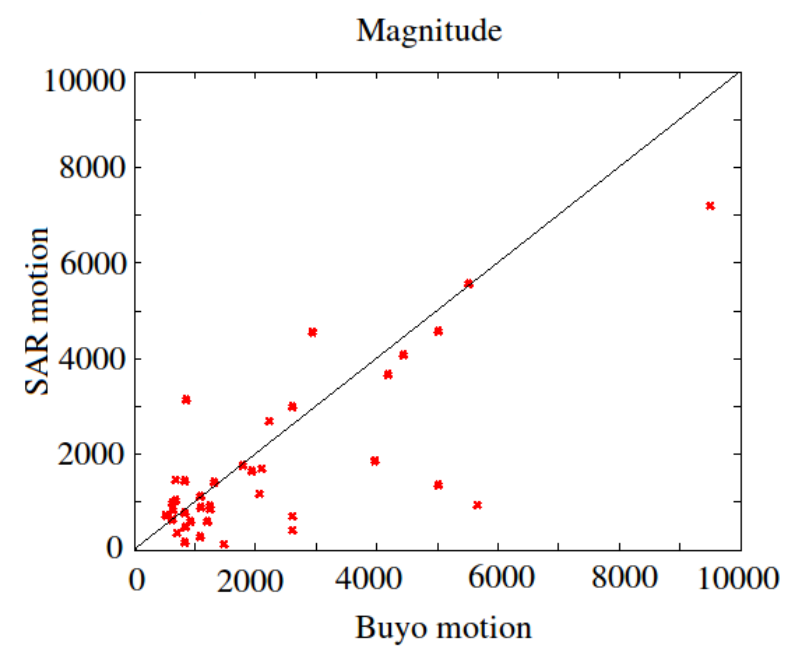

Fig. 8. Magnitude of the buoy motion and estimated motion for the long drift data in meters. One outlier with a buoy motion larger than the maximum algorithm detecting capability is located outside of the illustrated range.

against some buoy measurements. The algorithm had been in test use already in the previous ice season 2009-2010.

The algorithm produces reasonable values compared to the validation data from buoys. However, the validation data are currently only for the Gulf of Bothnia area, and no validation data for the Gulf of Finland exist. The ice drift direction is 
Direction

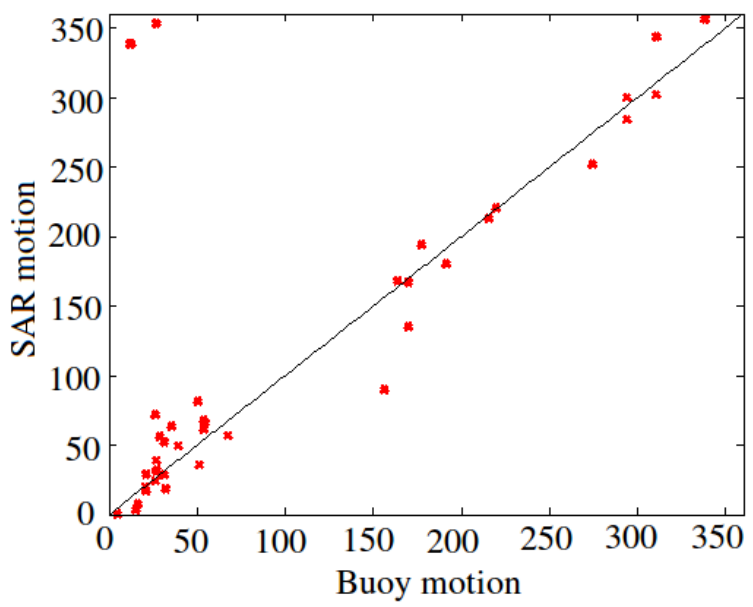

Fig. 9. Direction of the buoy motion and the estimated motion in degrees. The values correspond very well to each other. The high values of SAR drift directions for the low buoy drift directions are actually quite close to the buoy drift directions, because the direction 0 degrees (to north) corresponds to the value 360 degrees.

relatively well estimated for the long ice motion case; the accuracy in the magnitude is worse. For the short motion case, the estimated motion is also rather short, but the relative errors are rather high. The algorithm also reliably locates the areas where the ice is moving and the areas of static ice. These data can be used selectively in ice model validation. The data have not been used in data assimilation yet, but this is one potential application for the data. According to our experience, one very fruitful application for these data is SAR algorithms estimating the degree of ice deformation and locating fast ice, based on ice drift statistics for a period ranging from one week to a few weeks. The algorithm works well for SAR data in cold weather conditions. In the melting season, the ice cover gets wet and SAR backscattering is attenuated by the wet snow cover, and the sea ice feature detection becomes more difficult. This leads to poorer ice drift estimation algorithm performance in the wet snow conditions.

One restriction of the algorithm is related to the image size and resolution. Because the pixel size in the low resolution is $1.6 \mathrm{~km}$ and the window size is $16 \times 16$ pixels, it is not possible to correctly estimate motions larger than about $12.8 \mathrm{~km}$ in row or column directions, and $\sqrt{2} \times$ $12.8 \mathrm{~km}=18.1 \mathrm{~km}$ in diagonal directions. In practice, this limit is even smaller, because, at the window edges, the Gaussian window multiplication reduces the signal information content. This parametrization restricts the maximal allowed temporal difference between the two SAR images in the image pair. However, the window sizes and resolution are also user-defined parameters and easy to change, if e.g. longer al-

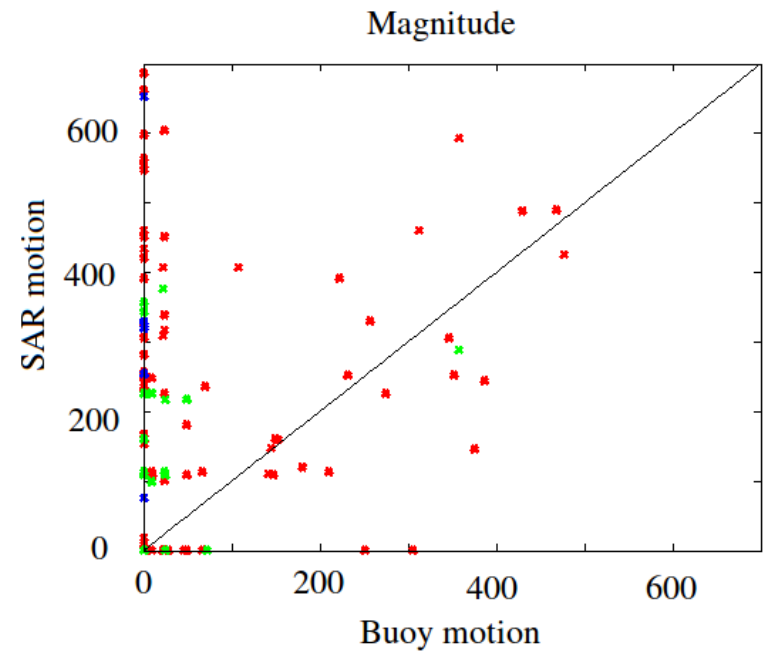

Fig. 10. Magnitude of the buoy drift and estimated ice drift for the short drift data in meters. The different quality classes are indicated by colors $\left(Q_{s}=2 \rightarrow\right.$ red, $Q_{s}=3 \rightarrow$ green, $Q_{s}=4 \rightarrow$ blue $)$.

lowed temporal differences between a SAR image pair acquisitions are required. However, the temporal difference cannot be increased very much, because with long temporal differences, more ice deformation occurs and the correlating features are lost.

Future improvements for the algorithm will be better taking into account ice rotations. In our coastal radar ice tracking algorithm (Karvonen, 2012), which is based on a similar phase correlation approach in two resolutions, we have taken this into account by computing the correlation for windows slightly rotated with respect to each other to the two directions in the low resolution, and using these rotations in the high-resolution motion candidates, i.e. it is not necessary to compute multiple correlations for different rotations in the high resolution. We are also going to adapt some image filtering techniques we have found useful in the coastal radar image ice tracking. Such techniques are computing the motion estimates only for locations with enough corner points, and removing small details by spatial edge preserving filtering techniques. Requiring corners reduces the errors caused by similarities along linear edges, and filtering of small details reduces the errors caused by these small features, which can be either SAR noise or actual small-scale ice features. Small-scale ice features can cause errors, because it is more likely that there exist multiple such features, and small-scale features also typically change faster than larger ones as the ice moves. We are also studying ways how these features can be taken into account in quality factor values. Currently, we can say that the quality is increased as a function of increased number of corner points, and decreased as a function of increased small-scale details. 
Acknowledgements. Thanks to FMI and MyOcean for funding of the work.

Edited by: P. Brasseur

\section{References}

Astola, J., Haavisto, P., and Neuvo, Y.: Vector Median Filters, Proc. of the IEEE, 78, 678-689, 1990.

Berg, A. and Eriksson, L. E. B.: Evaluation of a sea ice algorithm for SAR data from the Bay of Bothnia, Proc. of the third international workshop SeaSAR 2010, Frascati, Italy, ESA SP-679, 2010.

Biazzi, B., Alparone, L., Baronti, S., and Borri, G.: Pyramid-Based Multiresolution Adaptive Filters for Additive and Multiplicative Image Noise, IEEE T. Circuits Syst., 45, 1092-1097, 1998.

Canny, J.: A Computational Approach to Edge Detection, IEEE T. Pattern Anal., 8, 679-698, 1986.

Fily, M. and Rothrock, D. A.: Sea ice tracking by nested correlations, IEEE T. Geosci. Remote, GE-25, 5, 570-580, 1987.

Haarpaintner, J.: Arctic-wide operational sea ice drift from enhanced-resolution QuikScat/SeaWinds scatterometry and its validation, IEEE T. Geosci. Remote, 44, 102-107, 2006.

Horn, B. K. P. and Schunck, B. G.: Determining optical flow, Artificial Intelligence, 17, 185-203, 1981.

J. Karvonen, M. Simila, M. Makynen, Open Water Detection from Baltic Sea Ice Radarsat-1 SAR Imagery, IEEE T. Geosci. Remote, 2, 275-279, 2005.

J. Karvonen, B. Cheng, M. Simila, M. Hallikainen, Baltic Sea Ice Thickness Charts Based on Thermodynamic Snow/Ice Model, CBand SAR Classification and Ice Motion Detection, Proc. Int. Geosci. R. Sens. Symp., (IGARSS-08), 173-176, 2008.

Karvonen, J., Cheng, B., and Simila, M.: Ice Thickness Charts Produced by C-Band SAR Imagery and HIGHTSI Thermodynamic Ice Model, Proc. of the Sixth Workshop on Baltic Sea Ice Climate, 71-81, Lammi, Finland, 2008.

Karvonen, J.: Tracking of the Motion of Recognizable Sea Ice Objects from Coastal Radar Image Sequences, Annals of Glaciology, in review, 2012.
Kwok, R.: The RADARSAT Geophysical Processor System, in Analysis of SAR data of the Polar Oceans, in: Recent Advances, edited by: Tsatsoulis, C. and Kwok, R., 235-257, Springer Verlag, 1998.

Launiainen, J. and Cheng, B.: Modeling of ice thermodynamics in natural water Bodies, Cold Reg. Sci Technol., 27, 153-178, 1998.

Liu, A. K. and Cavalieri, D. J.: On sea ice drift from the wavelet analysis of the Defense Meteorological Satellite Program (DMSP) Special Sensor Microwave Imager (SSM/I) data, Int. J. Remote Sens., 19, 1415-1423, 1998.

Makynen, M., Manninen, T., Simila, M., Karvonen, J., and Hallikainen, M.: Incidence Angle Dependence of the Statistical Properties of the C-Band HH-Polarization Backscattering Signatures of the Baltic Sea Ice, IEEE T. Geosci. Remote, 40, 2593 2605, 2002.

MyOcean product catalogue at http://www.myocean.eu/web/ 24-catalogue.php, link checked July 8, 2012, 2012.

Sun, Y.: A new correlation technique for ice-motion analysis, EARSeL Adv. Remote Sens., 3, 57-63, 1994.

Sun, Y.: Automatic ice motion retrieval from ERS-1 SAR images using the optical flow method, Int. J. Remote Sens., 17, 20592087, 1996.

Thomas, M., Geiger, C., and Kambhamettu, C.: Discontinuous NonRigid Motion Analysis of Sea Ice using C-Band Synthetic Aperture Radar Satellite Imagery, IEEE Workshop on Articulated and Nonrigid Motion (ANM, In conjunction with CVPR'04), available at: http://vims.cis.udel.edu/sea/ice, 2004.

Thomas, M., Geiger, C. A., and Kambhamettu, C.: High resolution $(400 \mathrm{~m})$ motion characterization of sea ice using ERS-1 SAR imagery, Cold Regions Science and Technology, 52, 207-223, 2008. 